\title{
On the $p$ th moment estimates of solutions to stochastic functional differential equations in the G-framework
}

\author{
Faiz Faizullah ${ }^{*}$
}

*Correspondence:
faiz_math@ceme.nust.edu.pk
Department of Basic Sciences
and Humanities, College
of Electrical and Mechanical
Engineering, National
University of Sciences
and Technology (NUST),
Islamabad, Pakistan

${ }^{*}$ Correspondence: raiz_math@cemenustedupk and Humanities, College of Electrical and Mechanical Engineering, National and Technology (NUST) slamabad, Pakistan

\begin{abstract}
The aim of the current paper is to present the path-wise and moment estimates for solutions to stochastic functional differential equations with non-linear growth condition in the framework of G-expectation and G-Brownian motion. Under the nonlinear growth condition, the pth moment estimates for solutions to SFDEs driven by G-Brownian motion are proved. The properties of G-expectations, Hölder's inequality, Bihari's inequality, Gronwall's inequality and Burkholder-Davis-Gundy inequalities are used to develop the above mentioned theory. In addition, the path-wise asymptotic estimates and continuity of pth moment for the solutions to SFDEs in the G-framework, with non-linear growth condition are shown.
\end{abstract}

Keywords: $p$ th Moment estimates, G-Brownian motion, Stochastic functional differential equations, Path-wise asymptotic estimates, Non-linear growth condition

\section{Background}

Stochastic dynamical systems have a wide range of applications inside as well as outside the field of mathematics. The quantitative studies of different fields such as physics, engineering, ecological sciences, system sciences and medicine have been driven by stochastic dynamical systems. Stochastic differential equations (SDEs) are often used to model financial quantities such as asset prices, interest rates and their derivatives. These equations have become standard models for population dynamics and biological systems. Stochastic functional differential equations (SFDEs) in the G-framework were initiated by Ren et al. (2013). Then studied by Faizullah (2014), he developed the existence-and-uniqueness theorem with Cauchy-Maruyama approximation scheme (Faizullah 2014). Later, he proved the comparison result, with the help of which he established the existence theory for SFDEs in the G-framework with discontinuous drift coefficients (Faizullah et al. 2016). G-expectation, which is a nonlinear expectation, defined by Peng (2006), has been motivated by stochastic volatility problems and risk measures in finance (Gao 2009; Peng 2008, 2010). This led him to derive G-Brownian motion that is a novel stochastic process. Being different from the classical Brownian motion as it is not based on a given particular probability space, G-Brownian motion qualifies itself for a new and extremely rich structure which nontrivially generalizes the classical one. Some of the pertinent stochastic calculus

(c) 2016 The Author(s). This article is distributed under the terms of the Creative Commons Attribution 4.0 International License (http://creativecommons.org/licenses/by/4.0/), which permits unrestricted use, distribution, and reproduction in any medium, provided you give appropriate credit to the original author(s) and the source, provide a link to the Creative Commons license, and indicate if changes were made. 
which were established by him included G-Itô's integral, G-Itô’s formula and G-quadratic variation process $\langle B\rangle$. A new and interesting phenomenon that is related to the G-Brownian motion is the fact that its quadratic variation process, which is also a continuous process, has got stationary and independent increments. Therefore, it continues to qualify for being termed as a Brownian motion. Thus, the idea of G-framework-related stochastic differential equations was initiated (Peng 2006, 2008). Due to the applicability of the theory, many authors published their work on this emerging phenomenon in a short span of time (Bai and Lin 2014; Denis et al. 2010; Xua and Zhang 2009). As important as the existence theory, moment estimate is one of the most useful and basic schemes of analyzing dynamic behavior of SFDEs. It is also worth noting that the $p$ th moment of the solution for such SDEs driven by G-Brownian motion with non-linear growth condition has not been fully explored, which remains an interesting research topic. This article will fill the mentioned gap. We present the analysis for the solution to the following SFDE in the G-framework

$$
d Y(t)=\kappa\left(t, Y_{t}\right) d t+\lambda\left(t, Y_{t}\right) d\langle B, B\rangle(t)+\mu\left(t, Y_{t}\right) d B(t), \quad t \in[0, \infty),
$$

with initial data $Y_{t_{0}}=\zeta$ satisfying

$$
\begin{gathered}
Y_{t_{0}}=\zeta=\{\zeta(\theta):-\tau<\theta \leq 0\} \text { is } \mathcal{F}_{0} \text {-measurable, } B C\left([-\tau, 0] ; \mathbb{R}^{n}\right) \text {-valued } \\
\text { random variable such that } \zeta \in M_{G}^{2}\left([-\tau, 0] ; \mathbb{R}^{n}\right) .
\end{gathered}
$$

It is understood that $Y(t)$ is the value of stochastic process at time $t$ and $Y_{t}=\{Y(t+\theta):-\rho \leq \theta \leq 0, \rho>0\}$, indicates $B C([-\rho, 0] ; \mathbb{R})$-valued stochastic process, which is a collection of continuous and bounded real valued functions $\varphi$ defined on $[-\rho, 0]$ having norm $\|\varphi\|=\sup _{-\rho \leq \theta \leq 0}|\varphi(\theta)|$. The coefficients $\kappa, \lambda$ and $\mu$ are Borel measurable real valued functions on $[0, T] \times B C([-\rho, 0]$ (Faizullah et al. 2016). The rest of the paper is organized as follows: "Preliminaries" section is devoted to some basic definitions and results. " $p$ th Moment estimates for SFDEs in the G-framework" section presents the $p$ th moment estimates for SFDEs in the G-framework, under non-linear growth condition. "Continuity of $p$ th moment for SFDE in the G-framework" section shows that the $p$ th moment of solution to SFDE is continuous. The path-wise asymptotic estimates are given in "Path-wise asymptotic estimate" section.

\section{Preliminaries}

In this section some fundamental notions and results are given, which are used in the forthcoming sections of this paper. For more detailed literature of G-expectation, see the papers Denis et al. (2010), Faizullah (2012), Li and Peng (2011), Song (2013) and book Peng (2010).

Definition 1 Let $\mathcal{H}$ be a linear space of real valued functions defined on a nonempty basic space $\Omega$. Then a sub-linear expectation $E$ is a real valued functional on $\mathcal{H}$ with the following features:

(a) For all $Y, Z \in \mathcal{H}$, if $Y \leq Z$ then $E[Y] \leq E[Z]$.

(b) For any real constant $\gamma, E[\gamma]=\gamma$.

(c) For any $\theta>0, E[\theta Z]=\theta E[Z]$.

(d) For every $Y, Z \in \mathcal{H}, E[Y+Z] \leq E[Y]+E[Z]$. 
Let $C_{b . L i p}\left(\mathbb{R}^{l \times d}\right)$ denotes the set of bounded Lipschitz functions on $\mathbb{R}^{l \times d}$ and

$$
L_{G}^{p}\left(\Omega_{T}\right)=\left\{\phi\left(B_{t_{1}}, B_{t_{2}}, \ldots, B_{t_{l}} / l \geq 1, t_{1}, t_{2}, \ldots, t_{l} \in[0, T], \phi \in C_{b . L i p}\left(\mathbb{R}^{l \times d}\right)\right)\right\} .
$$

Let $\delta_{i} \in L_{G}^{p}\left(\Omega_{t_{i}}\right), i=0,1, \ldots, N-1$ then $M_{G}^{0}(0, T)$ denotes the collection of processes of the following type:

$$
\eta_{t}(w)=\sum_{i=0}^{N-1} \delta_{i}(w) I_{\left[t_{i}, t_{i+1}\right]}(t),
$$

where the above process is defined on a partition $\pi_{T}=\left\{t_{0}, t_{1}, \ldots, t_{N}\right\}$ of $[0, T]$. Associated with norm $\|\eta\|=\left\{\int_{0}^{T} E\left[\left|\eta_{u}\right|^{p}\right] d u\right\}^{1 / p}, M_{G}^{p}(0, T), p \geq 1$, is the completion of $M_{G}^{0}(0, T)$.

Definition 2 Let $\left(B_{t}\right)_{t \geq 0}$ be a $d$-dimensional stochastic process defined on $\left(\Omega, C_{l, l i p}(H), E\right)$, such that $B_{0}=0$. The increment $B_{t+m}-B_{t}$ is G-normally distributed for any $t, m \geq 0, n \in N$ and $0 \leq t_{1} \leq t_{2} \leq, \cdots, \leq t_{n} \leq t$, it is independent from $B_{t_{1}}, B_{t_{2}}, \ldots B_{t_{n}}$. Then $\left(B_{t}\right)_{t \geq 0}$ is known as G-Brownian motion.

For every $\eta_{t} \in M_{G}^{2,0}(0, T)$, the G-Itô's integral $I(\eta)$ and G-quadratic variation processes $\left\{\langle B\rangle_{t}\right\}_{t \geq 0}$ are respectively given by

$$
\begin{aligned}
& I(\eta)=\int_{0}^{T} \eta_{u} d B_{u}=\sum_{i=0}^{N-1} \delta_{i}\left(B_{t_{i+1}}-B_{t_{i}}\right), \\
& \langle B\rangle_{t}=B_{t}^{2}-2 \int_{0}^{t} B_{u} d B_{u} .
\end{aligned}
$$

We now state three important inequalities known as Hölder's inequality, Bihari's inequality and Gronwall's inequality respectively (Mao 1997).

Lemma 3 If $\frac{1}{q}+\frac{1}{r}=1$ for any $q, r>1, g \in L^{2}$ and $h \in L^{2}$ then $g h \in L^{1}$ and

$$
\int_{c}^{d} g h \leq\left(\int_{c}^{d}|g|^{q}\right)^{\frac{1}{q}}\left(\int_{c}^{d}|h|^{r}\right)^{\frac{1}{r}} .
$$

Lemma 4 Let $C \geq 0, h(t) \geq 0$ and $w(t)$ be a real valued continuous function on [c, $d]$. If for all $c \leq t \leq d, w(t) \leq C+\int_{c}^{d} h(s) w(s) d s$, then

$$
w(t) \leq C e^{\int_{c}^{t} h(s) d s},
$$

for all $c \leq t \leq d$.

The following two lemmas are borrowed from the book Mao (1997).

Lemma 5 Let $a, b \geq 0$ and $\epsilon \in(0,1)$. Then

$$
(a+b)^{2} \leq \frac{a^{2}}{\epsilon}+\frac{b^{2}}{1-\epsilon} .
$$


Lemma 6 Assume $p \geq 2$ and $\hat{\epsilon}, a, b>0$. Then the following two inequalities hold.

$$
\begin{aligned}
& \text { (i) } \quad a^{p-1} b \leq \frac{(p-1) \hat{\epsilon} a^{p}}{p}+\frac{b^{p}}{p \hat{\epsilon}^{p-1}} . \\
& \text { (ii) } \quad a^{p-2} b^{2} \leq \frac{(p-2) \hat{\epsilon} a^{p}}{p}+\frac{2 b^{p}}{p \hat{\epsilon}^{\frac{p-2}{2}}} \text {. }
\end{aligned}
$$

Theorem 7 Let $Y \in L^{p}$. Then for each $\epsilon>0$,

$$
\hat{C}\left(|Y|^{p}>\epsilon\right) \leq \frac{E\left[|Y|^{p}\right]}{\epsilon} .
$$

In the above Theorem 7, $\hat{C}$ is known as capacity defined by $\hat{C}(H)=\sup _{P \in \mathcal{P}} P(H)$, where $\mathcal{P}$ is a collection of all probability measures on $(\Omega, \mathcal{B}(\Omega)$ and $H \in \mathcal{B}(\Omega)$, which is Borel $\sigma$-algebra of $\Omega$. Also, we remind $\hat{C}(H)=0$ means that set $H$ is polar and a property holds quasi-surely (q.s. in short) means that it holds outside a polar set. The rest of the paper is organized as follows. In "Preliminaries" section, the pth moment estimates are studied. In " $p$ th Moment estimates for SFDEs in the G-framework" section, continuity of $p$ th moment is shown. In "Continuity of $p$ th moment for SFDE in the G-framework" section, path-wise asymptotic estimates for SFDEs driven by G-Brownian motion are given.

\section{pth Moment estimates for SFDEs in the G-framework}

Let Eq. (1) admit a unique solution $Y(t)$. Assume that a non-linear growth condition holds, which is given as follows. For every $\psi \in B C\left([-\tau, 0] ; \mathbb{R}^{d}\right)$ and $t \in[0, T]$,

$$
|\kappa(t, \psi)|^{2}+|\lambda(t, \psi)|^{2}+|\mu(t, \psi)|^{2} \leq \Upsilon\left(1+|\psi|^{2}\right)
$$

where $\Upsilon(\cdot): \mathbb{R}_{+} \rightarrow \mathbb{R}_{+}$is a non-decreasing and concave function such that $\Upsilon(0)=0, \Upsilon(x)>0$ for $x>0$ and

$$
\int_{0+} \frac{d x}{\Upsilon(x)}=\infty
$$

As $\Upsilon$ is concave and $\Upsilon(0)=0$, there exists two positive constants $\alpha$ and $\beta$ such that

$$
\Upsilon(x) \leq \alpha+\beta x,
$$

for all $x \geq 0$.

Theorem 8 Assume that the non-linear growth condition (3) holds. Let $E\|\zeta\|^{p}<\infty$ and $p \geq 2$. Then

$$
E\left[\sup _{-\tau \leq \nu \leq T}|Y(v)|^{p}\right] \leq E\|\zeta\|^{p}+\alpha_{3} e^{\beta_{3} T}
$$

where $\alpha_{3}=T\left[2 \alpha_{1}\left(1+c_{1}\right)+\alpha_{2}\left(c_{1}(p-1)+p c_{3}^{2}\right)\right], \beta_{3}=\left[2 \beta_{1}\left(1+c_{1}\right)+\beta_{2}\left(p-1+p c_{3}^{2}\right)\right]$, $\left.\alpha_{1}=\frac{1}{\hat{\epsilon}^{p-1}}(2)^{\frac{p}{2}-1}[(\alpha+\beta))^{\frac{p}{2}}+(\beta)^{\frac{p}{2}} E\|\zeta\|^{p}\right]$ and $\beta_{1}=(p-1) \hat{\epsilon}+\frac{(2 \beta)^{\frac{p}{2}}}{2 \hat{\epsilon}^{-1}}, \alpha_{2}=\frac{1}{\hat{\epsilon}^{\frac{p-2}{2}}}(2)^{\frac{p}{2}}$ $\left[(\alpha+\beta)^{\frac{p}{2}}+(\beta)^{\frac{p}{2}} E\|\zeta\|^{p}\right], \beta_{2}=\left[(p-1) \hat{\epsilon}+\frac{(2 \beta)^{\frac{p}{2}}}{\hat{\epsilon}^{\frac{p-2}{2}}}\right], c_{2}$ and $c_{3}$ are positive constants. 
Proof Applying G-Itôs formula to $|Y(t)|^{p}$, for $p \geq 2$, we proceed as follows

$$
\begin{aligned}
E\left[\sup _{0 \leq v \leq t}|Y(t)|^{p}\right] \leq & E|\zeta(0)|^{p}+p E\left[\sup _{0 \leq v \leq t} \int_{0}^{t}|Y(v)|^{p-1}\left|\kappa\left(v, Y_{v}\right)\right| d v\right] \\
& +E\left[\sup _{0 \leq v \leq t} \int_{0}^{t} p|Y(v)|^{p-1}\left|\mu\left(v, Y_{v}\right)\right| d B(v)\right] \\
& +E\left[\operatorname { s u p } _ { 0 \leq v \leq t } \int _ { 0 } ^ { t } \left[p|Y(v)|^{p-1}\left|\lambda\left(v, Y_{v}\right)\right|\right.\right. \\
& \left.\left.+\frac{p(p-1)}{2}|Y(v)|^{p-2}\left|\mu\left(v, Y_{v}\right)\right|^{2}\right] d\langle B, B\rangle(v)\right] \\
= & E|\zeta(0)|^{p}+I_{1}+I_{2}+I_{3}
\end{aligned}
$$

where

$$
\begin{aligned}
& I_{1}=p E\left[\sup _{0 \leq v \leq t} \int_{0}^{t}|Y(v)|^{p-1}\left|\kappa\left(v, Y_{v}\right)\right| d v\right], \\
& I_{2}=p E\left[\sup _{0 \leq v \leq t} \int_{0}^{t}|Y(v)|^{p-1}\left|\mu\left(v, Y_{v}\right)\right| d B(v)\right], \\
& I_{3}=p E\left[\sup _{0 \leq v \leq t} \int_{0}^{t}\left[|Y(v)|^{p-1}\left|\lambda\left(v, Y_{v}\right)\right|+\frac{(p-1)}{2}|Y(v)|^{p-2}\left|\mu\left(v, Y_{v}\right)\right|^{2}\right] d\langle B, B\rangle(v)\right] .
\end{aligned}
$$

By non-linear growth condition (3) and Lemma 6 , for any $\hat{\epsilon}>0$, we get

$$
\begin{aligned}
|Y(t)|^{p-1}\left|\kappa\left(t, Y_{t}\right)\right| & \leq \frac{(p-1) \hat{\epsilon}|Y(t)|^{p}}{p}+\frac{\left|\kappa\left(t, Y_{t}\right)\right|^{p}}{p \hat{\epsilon}^{p-1}} \\
& \leq \frac{(p-1) \hat{\epsilon}\|Y(t)\|^{p}}{p}+\frac{\left[\Upsilon\left(1+\left\|Y_{t}\right\|^{2}\right)\right]^{\frac{p}{2}}}{p \hat{\epsilon}^{p-1}} \\
& \leq \frac{(p-1) \hat{\epsilon}\|Y(t)\|^{p}}{p}+\frac{\left[\alpha+\beta\left(1+\left\|Y_{t}\right\|^{2}\right)\right]^{\frac{p}{2}}}{p \hat{\epsilon}^{p-1}} .
\end{aligned}
$$

Using the inequality $(a+b)^{p} \leq 2^{p-1}\left(a^{p}+b^{p}\right)$ and the fact $\sup _{-\tau \leq v \leq T}|Y(v)|^{p} \leq\|\zeta\|^{p}+$ $\sup _{0 \leq v \leq T}|Y(v)|^{p}$, we proceed as follows

$$
\begin{aligned}
|Y(t)|^{p-1}\left|\kappa\left(t, Y_{t}\right)\right| & \leq \frac{(p-1) \hat{\epsilon}\|Y(t)\|^{p}}{p}+\frac{\left[\alpha+\beta+\beta\left\|Y_{t}\right\|^{2}\right]^{\frac{p}{2}}}{p \hat{\epsilon}^{p-1}} \\
& \leq \frac{(p-1) \hat{\epsilon}\|Y(t)\|^{p}}{p}+\frac{(2)^{\frac{p}{2}-1}\left[(\alpha+\beta)^{\frac{p}{2}}+(\beta)^{\frac{p}{2}}\left\|Y_{t}\right\|^{p}\right]}{p \hat{\epsilon}^{p-1}} \\
& \leq \frac{(p-1) \hat{\epsilon}\|Y(t)\|^{p}}{p}+\frac{(2)^{\frac{p}{2}-1}\left[(\alpha+\beta)^{\frac{p}{2}}+(\beta)^{\frac{p}{2}}\|\zeta\|^{p}+(\beta)^{\frac{p}{2}}\|Y(t)\|^{p}\right]}{p \hat{\epsilon}^{p-1}} \\
& \leq \frac{(p-1) \hat{\epsilon}\|Y(t)\|^{p}}{p}+\frac{(2)^{\frac{p}{2}}\left[(\alpha+\beta)^{\frac{p}{2}}+(\beta)^{\frac{p}{2}}\|\zeta\|^{p}\right]+(2 \beta)^{\frac{p}{2}}\|Y(t)\|^{p}}{2 p \hat{\epsilon}^{p-1}} \\
& =\frac{(2)^{\frac{p}{2}}\left[(\alpha+\beta)^{\frac{p}{2}}+(\beta)^{\frac{p}{2}}\|\zeta\|^{p}\right]}{2 p \hat{\epsilon}^{p-1}}+\left[\frac{(p-1) \hat{\epsilon}}{p}+\frac{(2 \beta)^{\frac{p}{2}}}{2 p \hat{\epsilon}^{p-1}}\right]\|Y(t)\|^{p},
\end{aligned}
$$


which yields

$$
p E|Y(t)|^{p-1}\left|\kappa\left(t, Y_{t}\right)\right| \leq \alpha_{1}+\beta_{1} E\|Y(t)\|^{p},
$$

where $\alpha_{1}=\frac{\left.(2)^{\frac{p}{2}}[(\alpha+\beta))^{\frac{p}{2}}+(\beta)^{\frac{p}{2}} E\|\zeta\|^{p}\right]}{2 \hat{\epsilon}^{p-1}}$ and $\beta_{1}=(p-1) \hat{\epsilon}+\frac{(2 \beta)^{\frac{p}{2}}}{2 \hat{\epsilon}^{p-1}}$. In a similar fashion as above we get

$$
\begin{aligned}
& p|Y(t)|^{p-1}\left|\lambda\left(t, Y_{t}\right)\right| \leq \alpha_{1}+\beta_{1}\|Y(t)\|^{p}, \\
& p|Y(t)|^{p-1}\left|\mu\left(t, Y_{t}\right)\right| \leq \alpha_{1}+\beta_{1}\|Y(t)\|^{p} .
\end{aligned}
$$

Next by using Lemma 6, non-linear growth condition (3), inequality $(a+b)^{p} \leq 2^{p-1}\left(a^{p}+b^{p}\right)$ and the fact $\sup _{-\tau \leq v \leq T}|Y(v)|^{p} \leq\|\zeta\|^{p}+\sup _{0 \leq v \leq T}|Y(v)|^{p}$, we have

$$
\begin{aligned}
|Y(t)|^{p-2}\left|\mu\left(t, Y_{t}\right)\right|^{2} & \leq \frac{(p-2) \hat{\epsilon}|Y(t)|^{p}}{p}+\frac{2\left|\mu\left(t, Y_{t}\right)\right|^{p}}{p \hat{\epsilon}^{\frac{p-2}{2}}} \\
& \leq \frac{(p-2) \hat{\epsilon}\|Y(t)\|^{p}}{p}+\frac{2\left[\Upsilon\left(1+\left\|Y_{t}\right\|^{2}\right)\right]^{\frac{p}{2}}}{p \hat{\epsilon}^{\frac{p-2}{2}}} \\
& \leq \frac{(p-2) \hat{\epsilon}\|Y(t)\|^{p}}{p}+\frac{2\left[\alpha+\beta\left(1+\left\|Y_{t}\right\|^{2}\right)\right]^{\frac{p}{2}}}{p \hat{\epsilon}^{\frac{p-2}{2}}} \\
& \leq \frac{(p-2) \hat{\epsilon}\|Y(t)\|^{p}}{p}+\frac{2\left[\alpha+\beta+\beta\left\|Y_{t}\right\|^{2}\right]^{\frac{p}{2}}}{p \hat{\epsilon}^{\frac{p-2}{2}}} \\
& \leq \frac{(p-1) \hat{\epsilon}\|Y(t)\|^{p}}{p}+\frac{(2)^{\frac{p}{2}}\left[(\alpha+\beta)^{\frac{p}{2}}+(\beta)^{\frac{p}{2}}\left\|Y_{t}\right\|^{p}\right]}{p \hat{\epsilon}^{\frac{p-2}{2}}} \\
& \leq \frac{(p-2) \hat{\epsilon}\|Y(t)\|^{p}}{p}+\frac{(2)^{\frac{p}{2}}\left[(\alpha+\beta)^{\frac{p}{2}}+(\beta)^{\frac{p}{2}}\|\zeta\|^{p}+(\beta)^{\frac{p}{2}}\|Y(t)\|^{p}\right]}{p \hat{\epsilon}^{\frac{p-2}{2}}} \\
& \leq \frac{(p-2) \hat{\epsilon}\|Y(t)\|^{p}}{p}+\frac{(2)^{\frac{p}{2}}\left[(\alpha+\beta)^{\frac{p}{2}}+(\beta)^{\frac{p}{2}}\|\zeta\|^{p}\right]+(2 \beta)^{\frac{p}{2}}\|Y(t)\|^{p}}{p \hat{\epsilon}^{\frac{p-2}{2}}} \\
& =\frac{(2)^{\frac{p}{2}}\left[(\alpha+\beta)^{\frac{p}{2}}+(\beta)^{\frac{p}{2}}\|\zeta\|^{p}\right]}{p \hat{\epsilon}^{\frac{p-2}{2}}}+\left[\frac{(p-1) \hat{\epsilon}}{p}+\frac{(2 \beta)^{\frac{p}{2}}}{\left.p \hat{\epsilon}^{\frac{p-2}{2}}\right]\|Y(t)\|^{p},}\right.
\end{aligned}
$$

which gives

$$
p E|Y(t)|^{p-2}\left|\mu\left(t, Y_{t}\right)\right|^{2} \leq \alpha_{2}+\beta_{2} E\|Y(t)\|^{p},
$$
where $\alpha_{2}=\frac{\left.(2)^{\frac{p}{2}}[(\alpha+\beta))^{\frac{p}{2}}+(\beta)^{\frac{p}{2}} E\|\zeta\|^{p}\right]}{\hat{\epsilon}^{\frac{p-2}{2}}}$ and $\beta_{2}=\left[(p-1) \hat{\epsilon}+\frac{(2 \beta)^{\frac{p}{2}}}{\hat{\epsilon}^{\frac{p-2}{2}}}\right]$. Then $I_{1}$ can be written
as follows

$$
\begin{aligned}
I_{1} & =E\left[\sup _{0 \leq v \leq t} \int_{0}^{t} p|Y(v)|^{p-1}\left|\kappa\left(v, Y_{v}\right)\right| d v\right] \\
& \leq \int_{0}^{t}\left[\alpha_{1}+\beta_{1} E\|Y(t)\|^{p}\right] d v \\
& \leq \alpha_{1} T+\beta_{1} \int_{0}^{t} E\left(\|Y(v)\|^{p}\right) d v .
\end{aligned}
$$


By inequalities (8) and the Burkholder-Davis-Gundy (BDG) inequalities (Gao 2009), $I_{2}$ can be written as follows

$$
\begin{aligned}
I_{2} & =E\left[\sup _{0 \leq v \leq t} \mid \int_{0}^{t}\left[p|Y(v)|^{p-1}\left|\lambda\left(v, Y_{v}\right)\right|+\frac{p(p-1)}{2}|Y(v)|^{p-2}\left|\mu\left(v, Y_{v}\right)\right|^{2}\right] d\langle B, B\rangle(v)\right] \mid \\
& \leq c_{1} \int_{0}^{t}\left[p E|Y(v)|^{p-1}\left|\lambda\left(v, Y_{v}\right)\right|+\frac{p(p-1)}{2} E|Y(v)|^{p-2}\left|\mu\left(v, Y_{v}\right)\right|^{2}\right] d v \\
& \leq c_{1} \int_{0}^{t}\left[\alpha_{1}+\beta_{1} E\|Y(t)\|^{p}+\frac{(p-1)}{2}\left(\alpha_{2}+\beta_{2} E\|Y(t)\|^{p}\right)\right] d v \\
& \leq c_{1}\left(\alpha_{1}+\frac{1}{2}(p-1) \alpha_{2}\right) T+c_{1}\left(\beta_{1}+\frac{1}{2}(p-1) \beta_{2}\right) \int_{0}^{t} E\|Y(t)\|^{p} d v
\end{aligned}
$$

Next we use the BDG inequalities (Gao 2009), inequality (8), mean value theorem and the inequality $|a||b| \leq \frac{a^{2}}{2}+\frac{b^{2}}{2}$ as follows

$$
\begin{aligned}
I_{3} & =p E\left[\left.\sup _{0 \leq v \leq t}\left|\int_{0}^{t}\right| Y(v)\right|^{p-1}\left|\mu\left(v, Y_{v}\right)\right| d B(v) \mid\right] \\
& \leq p c_{3} E\left[\int_{0}^{t}|Y(v)|^{2 p-2}\left|\mu\left(v, Y_{v}\right)\right|^{2} d v\right]^{\frac{1}{2}} \\
& \leq p c_{3} E\left[\sup _{0 \leq v \leq t}\left[|Y(v)|^{p}\right]^{\frac{1}{2}} \int_{0}^{t}|Y(v)|^{p-2}\left|\mu\left(v, Y_{v}\right)\right|^{2} d v\right]^{\frac{1}{2}} \\
& \leq \frac{1}{2} E\left[\sup _{0 \leq v \leq t}|Y(v)|^{p}\right]+\frac{p^{2} c_{3}^{2}}{2} E\left[\int_{0}^{t}|Y(v)|^{p-2}\left|\mu\left(v, Y_{v}\right)\right|^{2} d v\right] \\
& \leq \frac{1}{2} E\left[\sup _{0 \leq v \leq t}|Y(v)|^{p}\right]+\frac{p c_{3}^{2}}{2} \int_{0}^{t}\left[\alpha_{2}+\beta_{2} E\|Y(t)\|^{p}\right] d v \\
& \leq \frac{1}{2} p c_{3}^{2} \alpha_{2} T+\frac{1}{2} E\left[\sup _{0 \leq v \leq t}|Y(v)|^{p}\right]+\frac{1}{2} p c_{3}^{2} \beta_{2} \int_{0}^{t} E\|Y(t)\|^{p} d v
\end{aligned}
$$

Using the values of $I_{1}, I_{2}$ and $I_{3}$ in (2) we get

$$
\begin{aligned}
E\left[\sup _{0 \leq v \leq t}|Y(v)|^{p}\right] \leq & \alpha_{1} T+\beta_{1} \int_{0}^{t} E\left(\|Y(v)\|^{p}\right) d v \\
& +c_{1}\left(\alpha_{1}+\frac{1}{2}(p-1) \alpha_{2}\right) T+c_{1}\left(\beta_{1}+\frac{1}{2}(p-1) \beta_{2}\right) \int_{0}^{t} E\|Y(t)\|^{p} d v \\
& +\frac{1}{2} p c_{3}^{2} \alpha_{2} T+\frac{1}{2} E\left[\sup _{0 \leq v \leq t}|Y(v)|^{p}\right]+\frac{1}{2} p c_{3}^{2} \beta_{2} \int_{0}^{t} E\|Y(t)\|^{p} d v \\
= & \frac{1}{2} E\left[\sup _{0 \leq v \leq t}|Y(v)|^{p}+T\left[\alpha_{1}\left(1+c_{1}\right)+\frac{1}{2} \alpha_{2}\left(c_{1}(p-1)+p c_{3}^{2}\right)\right]\right. \\
& +\left[\beta_{1}\left(1+c_{1}\right)+\frac{1}{2} \beta_{2}\left(p-1+p c_{3}^{2}\right)\right] \int_{0}^{t} E\left(\|Y(v)\|^{p}\right) d v
\end{aligned}
$$


simplification yields,

$$
\begin{aligned}
E\left[\sup _{0 \leq v \leq t}|Y(v)|^{p}\right] \leq & T\left[2 \alpha_{1}\left(1+c_{1}\right)+\alpha_{2}\left(c_{1}(p-1)+p c_{3}^{2}\right)\right] \\
& +\left[2 \beta_{1}\left(1+c_{1}\right)+\beta_{2}\left(p-1+p c_{3}^{2}\right)\right] \int_{0}^{t} E\left(\|Z(v)\|^{p}\right) d v .
\end{aligned}
$$

By the Gronwall's inequality

$$
E\left[\sup _{0 \leq v \leq t}|Y(v)|^{p}\right] \leq \alpha_{3} e^{\beta_{3} t},
$$

where $\alpha_{3}=T\left[2 \alpha_{1}\left(1+c_{1}\right)+\alpha_{2}\left(c_{1}(p-1)+p c_{3}^{2}\right)\right]$ and $\beta_{3}=\left[2 \beta_{1}\left(1+c_{1}\right)+\beta_{2}(p-1\right.$ $\left.\left.+p c_{3}^{2}\right)\right]$. By taking $t=T$, we have

$$
E\left[\sup _{0 \leq v \leq T}|Y(v)|^{p}\right] \leq \alpha_{3} e^{\beta_{3} T} .
$$

Noting the fact that $\sup _{-\tau \leq v \leq T}|Y(v)|^{p} \leq\|\zeta\|^{p}+\sup _{0 \leq v \leq T}|Y(v)|^{p}$, we proceed as follows

$$
\begin{aligned}
E\left[\sup _{-\tau \leq v \leq T}|Y(v)|^{p}\right] & \leq E\|\zeta\|^{p}+E\left[\sup _{0 \leq v \leq T}|Y(v)|^{p}\right] \\
& \leq E\|\zeta\|^{p}+\alpha_{3} e^{\beta_{3} T} .
\end{aligned}
$$

The proof is complete.

\section{Continuity of $p$ th moment for SFDE in the G-framework}

In the next theorem, under non-linear growth condition, it is shown that the $p$ th moment of the solution to SFDE in the G-framework (1) is continuous.

Theorem 9 Assume the non-linear growth condition (3) holds. Let $E\|\zeta\|^{p}<\infty$ and $p \geq 2$. Then

$$
E\left[|Y(t)-Y(s)|^{p}\right] \leq \gamma(t)(t-s)^{p},
$$

where $\gamma(t)=3^{\frac{3 p}{2}-2}\left(1+c_{2}+c_{3}\right)\left[\alpha^{\frac{p}{2}}+\beta^{\frac{p}{2}}+\beta^{\frac{p}{2}} E\|\zeta\|^{p}+\beta^{\frac{p}{2}} \alpha_{3} e^{\beta_{3} T}\right], c_{2}, c_{3}, \alpha, \beta, \alpha_{3}$ and $\beta_{3}$ are positive constants.

Proof By using the inequality $(a+b+c)^{p} \leq 3^{p-1}\left(a^{p}+b^{p}+c^{p}\right)$, Eq. (1) follows

$$
\begin{aligned}
|Y(t)-Y(s)|^{p}= & 3^{p-1}\left|\int_{s}^{t} \kappa\left(q, Y_{q}\right) d q\right|^{p}+3^{p-1}\left|\int_{s}^{t} \lambda\left(q, Y_{q}\right) d\langle B, B\rangle(q)\right|^{p} \\
& +3^{p-1}\left|\int_{s}^{t} \mu\left(q, Y_{q}\right) d B(q)\right|^{p} .
\end{aligned}
$$

Applying G-expectation on both sides, using the BDG inequalities (Gao 2009), Holder's inequality and non-linear growth condition, we proceed as follows 


$$
\begin{aligned}
E \mid & Y(t)-\left.Y(s)\right|^{p} \\
& \leq 3^{p-1}(t-s)^{p-1} E \int_{s}^{t}\left|\kappa\left(q, Y_{q}\right)\right|^{p} d q+3^{p-1} c_{2}(t-s)^{p-1} \int_{s}^{t}\left|\lambda\left(q, Y_{q}\right)\right|^{p} d q \\
& +3^{p-1} c_{3}(t-s)^{p-1} \int_{s}^{t}\left|\mu\left(q, Y_{q}\right)\right|^{p} d q \\
\leq & 3^{p-1}(t-s)^{p-1} E \int_{s}^{t}\left[\Upsilon\left(1+\left\|Y_{q}\right\|^{2}\right)\right]^{\frac{p}{2}} d q+3^{p-1} c_{2}(t-s)^{p-1} \int_{s}^{t}\left[\Upsilon\left(1+\left\|Y_{q}\right\|^{2}\right)\right]^{\frac{p}{2}} d q \\
& +3^{p-1} c_{3}(t-s)^{p-1} \int_{s}^{t}\left[\Upsilon\left(1+\left\|Y_{q}\right\|^{2}\right)\right]^{\frac{p}{2}} d q \\
= & 3^{p-1}(t-s)^{p-1}\left[1+c_{2}+c_{3}\right] E \int_{s}^{t}\left[\Upsilon\left(1+\left\|Y_{q}\right\|^{2}\right)\right]^{\frac{p}{2}} d q \\
\leq & 3^{p-1}(t-s)^{p-1}\left[1+c_{2}+c_{3}\right] E \int_{s}^{t}\left[\alpha+\beta\left(1+\left\|Y_{q}\right\|^{2}\right)\right]^{\frac{p}{2}} d q \\
\leq & 3^{p-1}(t-s)^{p-1}\left[1+c_{2}+c_{3}\right] E \int_{s}^{t}\left[\alpha+\beta+\beta\left\|Y_{q}\right\|^{2}\right]^{\frac{p}{2}} d q \\
\leq & 3^{p-1}(t-s)^{p-1}\left[1+c_{2}+c_{3}\right] 3^{\frac{p}{2}-1} \int_{s}^{t}\left[(\alpha)^{\frac{p}{2}}+(\beta)^{\frac{p}{2}}+(\beta)^{\frac{p}{2}} E\left\|Y_{q}\right\|^{p}\right] d q \\
\leq & 3^{p-1}(t-s)^{p-1}\left[1+c_{2}+c_{3}\right] 3^{\frac{p}{2}-1} \int_{s}^{t}\left[\alpha^{\frac{p}{2}}+\beta \beta^{\frac{p}{2}}+\beta \beta^{\frac{p}{2}} E\|\zeta\|^{p}+\beta \beta^{\frac{p}{2}} \quad \text { sup } E\|Y(r)\|^{p}\right] d r \\
\leq & 3^{\frac{3 p}{2}-2}(t-s)^{p}\left[1+c_{2}+c_{3}\right]\left[\alpha^{\frac{p}{2}}+\beta^{\frac{p}{2}}+\beta^{\frac{p}{2}} E\|\zeta\|^{p}\right] \\
& +3^{\frac{3 p}{2}-2}(t-s)^{p-1}\left[1+c_{2}+c_{3}\right] \beta^{\frac{p}{2}} \int_{s}^{t} \quad \sup _{0 \leq s \leq r \leq q} E\|Y(r)\|^{p} d r \\
&
\end{aligned}
$$

By using the inequality (11), it follows

$$
\begin{aligned}
E|Y(t)-Y(s)|^{p} \leq & 3^{\frac{3 p}{2}-2}(t-s)^{p}\left[1+c_{2}+c_{3}\right]\left[\alpha^{\frac{p}{2}}+\beta^{\frac{p}{2}}+\beta^{\frac{p}{2}} E\|\zeta\|^{p}\right] \\
& +3^{\frac{3 p}{2}-2}(t-s)^{p-1}\left[1+c_{2}+c_{3}\right] \beta^{\frac{p}{2}} \int_{s}^{t} \alpha_{3} e^{\beta_{3} T} d r \\
\leq & 3^{\frac{3 p}{2}-2}(t-s)^{p}\left[1+c_{2}+c_{3}\right]\left[\alpha^{\frac{p}{2}}+\beta^{\frac{p}{2}}+\beta^{\frac{p}{2}} E\|\zeta\|^{p}\right] \\
& +3^{\frac{3 p}{2}-2}(t-s)^{p}\left[1+c_{2}+c_{3}\right] \beta^{\frac{p}{2}} \alpha_{3} e^{\beta_{3} T} \\
= & \gamma(t)(t-s)^{p},
\end{aligned}
$$

where $\gamma(t)=3^{\frac{3 p}{2}-2}\left(1+c_{2}+c_{3}\right)\left[\left[\alpha^{\frac{p}{2}}+\beta^{\frac{p}{2}}+\beta^{\frac{p}{2}} E\|\zeta\|^{p}+\beta^{\frac{p}{2}} \alpha_{3} e^{\beta_{3} T}\right]\right.$. The proof is complete.

In the above theorem $c_{2}, c_{3}, \alpha, \beta, \alpha_{3}$ and $\beta_{3}$ are positive constants. The values of $\alpha_{3}$ and $\beta_{3}$ are given in Theorem 8 .

\section{Path-wise asymptotic estimate}

Next, by using Theorem 8 we study the path-wise asymptotic estimate for the solution of SFDE in the G-framework (1). It is understood that $\lim _{t \rightarrow \infty} \sup \frac{1}{t} \log |Y(t)|$ is the Lyapunov exponent (Kim 2014). It is shown that the $p$ th moment of Lyapunov exponent should not be greater than $\frac{1}{p}\left[2 \beta_{1}\left(1+c_{1}\right)+\beta_{2}\left(p-1+p c_{3}^{2}\right)\right]$, where $c_{1}, c_{3}, \beta_{1}, \beta_{2}$ are positive constants and $p \geq 2$. 
Theorem 10 Assume that the non-linear growth condition (3) holds. Then

$$
\lim _{t \rightarrow \infty} \sup \frac{1}{t} \log |Y(t)| \leq \frac{1}{p}\left[2 \beta_{1}\left(1+c_{1}\right)+\beta_{2}\left(p-1+p c_{3}^{2}\right)\right] \quad \text { q.s. }
$$

Proof For each $k=1,2, \ldots$, using the non-linear growth condition in a similar fashion as in Theorem 8, Eq. (10) we obtain,

$$
E\left(\sup _{k-1 \leq t \leq k}|Y(t)|^{p}\right) \leq \alpha_{3} e^{\beta_{3} k}
$$

where $\alpha_{3}=T\left[2 \alpha_{1}\left(1+c_{1}\right)+\alpha_{2}\left(c_{1}(p-1)+p c_{3}^{2}\right)\right]$ and $\beta_{3}=\left[2 \beta_{1}\left(1+c_{1}\right)+\beta_{2}(p-1\right.$ $\left.\left.+p c_{3}^{2}\right)\right]$. Recall that $E$ is a sub-linear expectation. Unlike a classical expectation, it is not based on a particular probability space. So, instead of probability, we use a different concept known as capacity. Thanks to Theorem 7 for any arbitrary $\epsilon>0$, we have

$$
\begin{aligned}
\hat{C}\left(w: \sup _{k-1 \leq t \leq k}|Y(t)|^{p}>e^{\left(\beta_{3}+\epsilon\right) k}\right) & \leq \frac{E\left[\sup _{k-1 \leq t \leq k}|Y(t)|^{p}\right]}{e^{\left(\beta_{3}+\epsilon\right) k}} \\
& \leq \frac{\alpha e^{\beta_{3} k}}{e^{\left(\beta_{3}+\epsilon\right) k}} \\
& =\alpha e^{-\epsilon k} .
\end{aligned}
$$

The Borel-Cantelli lemma follows for almost all $w \in \Omega$, there exists a random integer $k_{0}=k_{0}(w)$ such that

$$
\sup _{k-1 \leq t \leq k}|Y(t)|^{p} \leq e^{\left(\beta_{3}+\epsilon\right) k} \quad \text { whenever } k \geq k_{0},
$$

consequently, we get

$$
\begin{aligned}
\lim _{t \rightarrow \infty} \sup \frac{1}{t} \log |Y(t)| & \leq \frac{\beta_{3}+\epsilon}{p} \\
& =\frac{1}{p}\left[2 \beta_{1}\left(1+c_{1}\right)+\beta_{2}\left(p-1+p c_{3}^{2}\right)\right]+\frac{\epsilon}{p}, \quad \text { q.s. }
\end{aligned}
$$

But $\epsilon$ is arbitrary, so

$$
\lim _{t \rightarrow \infty} \sup \frac{1}{t} \log |Y(t)| \leq \frac{1}{p}\left[2 \beta_{1}\left(1+c_{1}\right)+\beta_{2}\left(p-1+p c_{3}^{2}\right)\right], \text { q.s. }
$$

The proof is complete.

Remark 11 In the above theorem if $p=2$, then

$$
\lim _{t \rightarrow \infty} \sup \frac{1}{t} \log |Y(t)| \leq \beta_{1}\left(1+c_{1}\right)+\frac{1}{2} \beta_{2}\left(1+2 c_{3}^{2}\right),
$$

Hence $\beta_{1}\left(1+c_{1}\right)+\frac{1}{2} \beta_{2}\left(1+2 c_{3}^{2}\right)$ is the upper bound for second moment of Lyapunov exponent. 


\section{Conclusion}

Generally, we cannot find explicit solutions to nonlinear SDEs. Thus one needs to present the analysis for solutions to these equations. Existence and moment estimates are the most important characteristics for solutions to SDEs. Here, we have used some important inequalities such as Bihari's inequality, Hölder's inequality, Gronwall's inequality and Burkholder-Davis-Gundy (BDG) inequalities to investigate the $p$ th moment estimates for SFDEs driven by G-Brownian motion. Then the asymptotic estimates for these equations have been developed. Furthermore, continuity of $p$ th moment for the solutions to SFDEs in the G-framework has been proved. The G-Brownian motion theory is the generalization of the classical Brownian motion theory. The methodology used to estimate $p$ th moment for $\mathrm{SDE}$ is interesting and applicable in various practical applications. For example, $p$ th moment estimates are useful in biological population models (Shang 2013a) and distributed system control (Shang 2012, 2013b, 2015). The methods of the $p$ th moment estimation, developed in our paper, can be used to extend the related theory in above mentioned papers.

\section{Acknowledgements}

The author acknowledges and appreciates the financial support of NUST research directorate for this research work. We are very grateful to the anonymous reviewer for his/her useful suggestions, which have improved the quality of this paper.

Competing interests

The author declares that he has no competing interests.

Received: 6 May 2016 Accepted: 23 May 2016

Published online: 24 June 2016

\section{References}

Bai X, Lin Y (2014) On the existence and uniqueness of solutions to stochastic differential equations driven by G-Brownian motion with integral-Lipschitz coefficients. Acta Math Appl Sin 3(30):589-610

Denis L, Hu M, Peng S (2010) Function spaces and capacity related to a sublinear expectation: application to G-Brownian motion paths. Potential Anal 34:139-161

Faizullah F (2012) A note on the caratheodory approximation scheme for stochastic differential equations under G-Brownian motion. Z Naturforschung A 67a:699-704

Faizullah F (2014) Existence of solutions for G-SFDEs with Cauchy-Maruyama approximation scheme. Appl Anal Abstr. doi:10.1155/2014/809431

Faizullah F, Mukhtar A, Rana MA (2016) A note on stochastic functional differential equations driven by G-Brownian motion with discontinuous drift coefficients. J Comput Anal Appl 5(21):910-919

Gao F (2009) Pathwise properties and homeomorphic flows for stochastic differential equations driven by G-Brownian motion. Stoch Proc Appl 2:3356-3382

Kim YH (2014) On the pth moment estimates for the solution of stochastic differential equations. J Inequal Appl 395:1-9 Li X, Peng S (2011) Stopping times and related Ito's calculus with G-Brownian motion. Stoch Proc Appl 121:1492-1508

Mao X (1997) Stochastic differential equations and their applications. Horwood Publishing Chichester, Coll House England

Peng S (2006) G-expectation, G-Brownian motion and related stochastic calculus of Ito's type. In: The abel symposium 2 , Springer, Berlin, pp 541-567

Peng S (2008) Multi-dimentional G-Brownian motion and related stochastic calculus under G-expectation. Stoch Proc Appl 12:2223-2253

Peng S (2010) Nonlinear expectations and stochastic calculus under uncertainty. arXiv:1002.4546v1

Ren Y, Bi Q, Sakthivel R (2013) Stochastic functional differential equations with infinite delay driven by G-Brownian motion. Math Method Appl Sci 36(13):1746-1759

Shang Y (2012) Synchronization in networks of coupled harmonic oscillators with stochastic perturbation and time delays. Ann Acad Rom Sci Ser Math Appl 1(4):44-58

Shang Y (2013a) The limit behavior of a stochastic logistic model with individual time-dependent rates. J Math. doi:10.1155/2013/502635

Shang Y (2013b) Group consensus of multi-agent systems in directed networks with noises and time delays. Int J Syst Sci 14(46):2481-2492

Shang Y (2015) Consensus of noisy multiagent systems with Markovian switching topologies and time-varying delays. Math Probl Eng. doi:10.1155/2015/453072

Song Y (2013) Properties of hitting times for G-martingale and their applications. Stoch Process Appl 8(121):1770-1784

Xua J, Zhang B (2009) Martingale characterization of G-Brownian motion. Stoch Process Appl 119:232-248 